\title{
A Multilevel Correction Scheme for the Steklov Eigenvalue Problem
}

\author{
Qichao Zhao, Yidu Yang, and Hai Bi \\ School of Mathematics and Computer Science, Guizhou Normal University, Guiyang 550001, China \\ Correspondence should be addressed to Hai Bi; bihaimath@gznu.edu.cn
}

Received 5 May 2015; Accepted 2 August 2015

Academic Editor: John D. Clayton

Copyright (C) 2015 Qichao Zhao et al. This is an open access article distributed under the Creative Commons Attribution License, which permits unrestricted use, distribution, and reproduction in any medium, provided the original work is properly cited.

Combining the correction technique proposed by Lin and Xie and the shifted inverse iteration, a multilevel correction scheme for the Steklov eigenvalue problem is proposed in this paper. The theoretical analysis and numerical experiments indicate that the scheme proposed in this paper is efficient for both simple and multiple eigenvalues of the Steklov eigenvalue problem.

\section{Introduction}

Steklov eigenvalue problems have important applications in physics and engineering, for instance, in the study of surface waves (see [1]), in the analysis of stability of mechanical oscillators immersed in a viscous fluid (see [2]), and in the study of the vibration modes of a structure in contact with an incompressible fluid (see [3]). Thus, finite element methods for Steklov eigenvalue problems have attracted the attention of mathematics and physics community. Reference [4] first studied the convergence and error estimation of finite element approximations and [5-8] made in-depth research; after that, [9-15] discussed the highly efficient finite element methods.

Recently, an efficient multilevel method based on the correction step was proposed by Lin and Xie $[16,17]$ and further successfully applied to Helmholtz transmission eigenvalue problems [18], convection-diffusion eigenvalue problems [19], and the Steklov problem [15]. The multilevel correction method proposed by Lin and Xie can be regarded as a combination of two-grid method and the extended/generalized finite element method which was developed in 1990s (see $[20,21])$.

The shifted inverse iteration method is a basic approach for solving matrix eigenvalue problems (see Algorithm 27.3 in [22]). Now, two-grid methods based on the shifted inverse iteration have been established (see $[23,24])$ and applied to Steklov eigenvalue problems (see [11]).
In this paper, we combine the correction technique of Lin and Xie and the shifted inverse iteration to establish a new efficient multilevel scheme for the Steklov eigenvalue problem which is suitable not only for simple eigenvalues but also for multiple eigenvalues. Our scheme can be described as follows: (1) solve the Steklov eigenvalue problem in the coarsest finite element space; (2) implement the shifted inverse iteration once in an augmented space by using the previously obtained eigenvalue as the shift and eigenfunction as the iteration initial value; (3) solve the Steklov eigenvalue problem again in a new space which is constructed by combining the coarsest finite element space with the obtained eigenfunction approximation in (2), and then return to (2) for next loop. Furthermore, we prove the scheme can reach the optimal order that the same as solving the corresponding boundary value problem. Our scheme is easy to realize under the package of iFEM [25] with Matlab, and the numerical results are satisfactory.

In this paper, $C$ (with or without subscripts) denotes a positive constant independent of mesh diameters and correction times.

\section{Preliminaries}

Let $H^{s}(\Omega)$ and $H^{s}(\partial \Omega)$ denote the usual Sobolev spaces with real-order $s$ with norms $\|\cdot\|_{s}$ and $\|\cdot\|_{s, \partial \Omega}$ on $\Omega$ and $\partial \Omega$, respectively. Here $H^{0}(\partial \Omega)=L^{2}(\partial \Omega)$. 
Consider the following Steklov eigenvalue problem:

$$
\begin{aligned}
-\Delta u+u & =0, \quad \text { in } \Omega, \\
\frac{\partial u}{\partial v} & =\lambda u, \quad \text { on } \partial \Omega,
\end{aligned}
$$

where $\Omega \subset R^{2}$ is a bounded polygonal domain with the maximum interior angle $\omega$ and $\partial u / \partial \nu$ is the outward normal derivative on $\partial \Omega$.

The variational form of (1) is given as follows: find $\lambda \in R$ and $0 \neq u \in H^{1}(\Omega)$ such that $a(u, u)=1$ and

$$
a(u, v)=\lambda b(u, v), \quad \forall v \in H^{1}(\Omega),
$$

where

$$
\begin{aligned}
& a(u, v)=\int_{\Omega} \nabla u \cdot \nabla v+u v d x \\
& b(u, v)=\int_{\partial \Omega} u v d s .
\end{aligned}
$$

It is easy to know that $a(\cdot, \cdot)$ is a symmetric, continuous, and $H^{1}(\Omega)$-elliptic bilinear form on $H^{1}(\Omega) \times H^{1}(\Omega)$ satisfying

$$
\begin{aligned}
& a(w, v) \leq C_{1}\|w\|_{1}\|v\|_{1}, \quad \forall w, v \in H^{1}(\Omega), \\
& a(w, w) \geq c_{1}\|w\|_{1}^{2}, \quad \forall w \in H^{1}(\Omega) .
\end{aligned}
$$

So we can use $a(\cdot, \cdot)$ and $\|\cdot\|_{a}=a(\cdot, \cdot)^{1 / 2}$ as the inner product and norm on $H^{1}(\Omega)$, respectively. We can also use $b(\cdot, \cdot)$ and $\|\cdot\|_{b}=b(\cdot, \cdot)^{1 / 2}$ as the inner product and norm on $L^{2}(\partial \Omega)$, respectively.

From $[4,26]$ we know that problem (2) has an eigenvalue sequence $\left\{\lambda_{j}\right\}_{1}^{\infty}$ satisfying

$$
0<\lambda_{1} \leq \lambda_{2} \leq \cdots \leq \lambda_{j} \leq \cdots, \quad \lim _{j \rightarrow \infty} \lambda_{j}=\infty
$$

and the corresponding eigenfunctions

$$
u_{1}, u_{2}, \ldots, u_{j}, \ldots
$$

where $a\left(u_{i}, u_{j}\right)=\delta_{i j}$. The eigenvalues $\lambda_{j}$ are repeated according to their multiplicity in the sequence $\left\{\lambda_{j}\right\}_{1}^{\infty}$.

Let $\mathscr{T}_{h}$ be a regular triangulation of $\Omega$. Denote the diameter of an element $K \in \mathscr{T}_{h}$ by $h_{K}$ and the mesh diameter $h=\max _{K \in \mathscr{T}_{h}} h_{K}$. Let the finite element space $V_{h} \subset H^{1}(\Omega)$ be a piecewise polynomial space on $\mathscr{T}_{h}$.

Then the finite element approximation of (2) is given as follows: find $\lambda_{h} \in R$ and $0 \neq u_{h} \in V_{h}$ such that $\left\|u_{h}\right\|_{a}=1$ and

$$
a\left(u_{h}, v_{h}\right)=\lambda_{h} b\left(u_{h}, v_{h}\right), \quad \forall v_{h} \in V_{h} .
$$

It is well known that (7) has eigenvalues (see, e.g., $[4,26]$ )

$$
\begin{array}{r}
0<\lambda_{1, h} \leq \lambda_{2, h} \leq \cdots \leq \lambda_{j, h} \leq \cdots \leq \lambda_{N_{h}, h} \\
\\
\quad\left(N_{h}=\operatorname{dim} V_{h}\right),
\end{array}
$$

and the associated eigenfunctions

$$
u_{1, h}, u_{2, h}, \ldots, u_{j, h}, \ldots, u_{N_{h}, h}
$$

where $a\left(u_{i, h}, u_{j . h}\right)=\delta_{i j}, 1 \leq i, j \leq N_{h}$.

Consider the following boundary value problems (10) and

(11) associated with (2) and (7), respectively.

Find $\psi \in H^{1}(\Omega)$ such that $\|\psi\|_{a}=1$ and

$$
a(\psi, v)=b(f, v), \quad \forall v \in H^{1}(\Omega) .
$$

Find $\psi_{h} \in V_{h}$ such that $\left\|\psi_{h}\right\|_{a}=1$ and

$$
a\left(\psi_{h}, v_{h}\right)=b\left(f, v_{h}\right), \quad \forall v_{h} \in V_{h} .
$$

The following regularities of the Steklov eigenvalue problem are valid.

Lemma 1. If $f \in L^{2}(\partial \Omega)$, there exists a unique solution $\psi \in$ $H^{1+r / 2}(\Omega)$ to (10) satisfying

$$
\|\psi\|_{1+r / 2} \leq C_{2}\|f\|_{0, \partial \Omega} .
$$

If $f \in H^{1 / 2}(\partial \Omega)$, there exists a unique solution $\psi \in H^{1+r}(\Omega)$ to (10) satisfying

$$
\|\psi\|_{1+r} \leq C_{2}\|f\|_{1 / 2, \partial \Omega}
$$

where $C_{2}$ is a positive constant.

Proof. See (4.10) in [27], Proposition 4.4 in [3], and Lemma 2.1 in [11].

By (13), we know that $u_{j} \in H^{1+r}(\Omega), r=1$, if $\Omega$ is convex; otherwise $r<\pi / \omega$ and $r$ can be closed to $\pi / \omega$ arbitrarily.

Then, from (10) and (11), we can define two linear bounded operators $T: L^{2}(\partial \Omega) \rightarrow H^{1}(\Omega)$ and $T_{h}: L^{2}(\partial \Omega) \rightarrow V_{h}$ such that

$$
\begin{aligned}
a(T f, v) & =b(f, v), \quad \forall v \in H^{1}(\Omega), \\
a\left(T_{h} f, v_{h}\right) & =b\left(f, v_{h}\right), \quad \forall v_{h} \in V_{h} .
\end{aligned}
$$

It is obvious that $T f$ and $T_{h} f$ are the solution of (10) and (11), respectively. We know that $T: H^{1}(\Omega) \rightarrow H^{1}(\Omega)$ and $T_{h}: H^{1}(\Omega) \rightarrow V_{h}$ are completely continuous operators (see, e.g., [11]). From $[4,26]$, we know that (2) and (7) have the following equivalent operator forms:

$$
\begin{gathered}
T u=\mu u, \\
T_{h} u_{h}=\mu_{h} u_{h},
\end{gathered}
$$

where $\mu=1 / \lambda$ and $\mu_{h}=1 / \lambda_{h}$. From (15), we can deduce that $T_{h}: L^{2}(\partial \Omega) \rightarrow V_{h}$ is bounded; that is,

$$
\begin{aligned}
& \left\|T_{h} f\right\|_{a} \leq C_{3}\|f\|_{b}, \quad \forall f \in L^{2}(\partial \Omega), \\
& \left\|T_{h} f\right\|_{a} \leq C_{3}\|f\|_{a}, \quad \forall f \in H^{1}(\Omega),
\end{aligned}
$$

where $C_{3}$ is a positive constant independent of $h$ and $f$. 
Denote the eigenfunction space corresponding to $\lambda_{i}$ by

$$
\begin{aligned}
& M\left(\lambda_{i}\right)=\{w \\
& \quad \in H^{1}(\Omega) \mid w \text { is an eigenfunction of }(2) \text { corresponding to } \lambda_{i} \text { and }\|w\|_{a} \\
& =1\} .
\end{aligned}
$$

Suppose that the multiplicity of $\lambda_{i}$ is $q$; that is, $\lambda_{i}=\lambda_{i+1}=$ $\cdots=\lambda_{i+q-1}$. We use $\left(\lambda_{j, h}, u_{j, h}\right)$ to denote the eigenpair approximation for $\left(\lambda_{j}, u_{j}\right), j=i, \ldots, i+q-1$. Let

$$
M_{h}\left(\lambda_{i}\right)=\operatorname{span}\left\{u_{i, h}, u_{i+1, h}, \ldots, u_{i+q-1, h}\right\} \text {, }
$$

and let $M\left(\mu_{i}\right)=M\left(\lambda_{i}\right)$ and $M_{h}\left(\mu_{i}\right)=M_{h}\left(\lambda_{i}\right)$.

Denote

$$
\begin{gathered}
\eta_{a}(h)=\sup _{f \in L^{2}(\partial \Omega),\|f\|_{0, \partial \Omega}=1} \inf _{v_{h} \in V_{h}}\left\|T f-v_{h}\right\|_{a}, \\
\delta_{h}\left(\lambda_{i}\right)=\sup _{w \in M\left(\lambda_{i}\right)} \inf _{v_{h} \in V_{h}}\left\|w-v_{h}\right\|_{a} .
\end{gathered}
$$

From Lemma 1 and the interpolation error estimate, we get

$$
\eta_{a}(h) \longrightarrow 0, \quad \text { as } h \longrightarrow 0 \text {. }
$$

For two linear spaces $A$ and $B$, we define

$$
\begin{aligned}
\operatorname{dist}(u, A) & =\inf _{v \in A}\|u-v\|_{a}, \quad \forall u \in B, \\
\widehat{\Theta}(A, B) & =\sup _{w \in A,\|w\|_{a}=1} \inf _{v \in B}\|w-v\|_{a}, \\
\widehat{\Phi}(A, B) & =\sup _{w \in A,\|w\|_{b}=1} \inf _{v \in B}\|w-v\|_{b} .
\end{aligned}
$$

Define the gaps between $M\left(\lambda_{i}\right)$ and $M_{h}\left(\lambda_{i}\right)$ in $\|\cdot\|_{a}$ as

$$
\begin{aligned}
& \Theta\left(M\left(\lambda_{i}\right), M_{h}\left(\lambda_{i}\right)\right) \\
& =\max \left\{\widehat{\Theta}\left(M\left(\lambda_{i}\right), M_{h}\left(\lambda_{i}\right)\right), \widehat{\Theta}\left(M_{h}\left(\lambda_{i}\right), M\left(\lambda_{i}\right)\right)\right\}
\end{aligned}
$$

and in $\|\cdot\|_{b}$ as

$$
\begin{aligned}
& \Phi\left(M\left(\lambda_{i}\right), M_{h}\left(\lambda_{i}\right)\right)=\max \left\{\widehat{\Phi}\left(M\left(\lambda_{i}\right), M_{h}\left(\lambda_{i}\right)\right),\right. \\
& \left.\widehat{\Phi}\left(M_{h}\left(\lambda_{i}\right), M\left(\lambda_{i}\right)\right)\right\} .
\end{aligned}
$$

Lemma 2. The following estimates are valid:

$$
\begin{gathered}
\Theta\left(M\left(\lambda_{i}\right), M_{h}\left(\lambda_{i}\right)\right) \leq C\left(\lambda_{i}\right) \delta_{h}\left(\lambda_{i}\right), \\
\Phi\left(M\left(\lambda_{i}\right), M_{h}\left(\lambda_{i}\right)\right) \leq C\left(\lambda_{i}\right) \eta_{a}(h) \delta_{h}\left(\lambda_{i}\right), \\
\left|\lambda_{j, h}-\lambda_{i}\right| \leq C\left(\lambda_{i}\right) \delta_{h}^{2}\left(\lambda_{i}\right),
\end{gathered}
$$

for $j=i, \ldots, i+q-1$. Here and hereafter $C\left(\lambda_{i}\right)$ is a positive constant depending on $\lambda_{i}$ but independent of $h$.

Proof. See [4] or P. 699 in [26].

We need the following result (cf. $[11,23])$ for the shifted inverse iteration method.
Lemma 3. Let $\left(\mu_{0}, u_{0}\right)$ be an approximation for $\left(\mu_{i}, u_{i}\right)$, where $\mu_{0}$ is not an eigenvalue of $T_{h}$, and $u_{0} \in V_{h}$ with $\left\|u_{0}\right\|_{a}=1$. Suppose that $\operatorname{dist}\left(u_{0}, M_{h}\left(\mu_{i}\right)\right) \leq 1 / 2,\left|\mu_{0}-\mu_{j, h}\right| \geq \rho / 2$ for $j \neq$ $i, \ldots, i+q-1$, and $u \in V_{h}, u_{j}^{h} \in V_{h}$ satisfy

$$
\begin{aligned}
\left(\mu_{0}-T_{h}\right) u & =u_{0}, \\
u_{j}^{h} & =\frac{u}{\|u\|_{a}} .
\end{aligned}
$$

Then

$$
\begin{aligned}
& \operatorname{dist}\left(u_{j}^{h}, M_{h}\left(\lambda_{i}\right)\right) \\
& \leq C_{4}\left(\left|\lambda_{0}-\lambda_{i}\right|+C\left(\lambda_{i}\right) \delta_{h}^{2}\left(\lambda_{i}\right)\right) \operatorname{dist}\left(u_{0}, M_{h}\left(\lambda_{i}\right)\right),
\end{aligned}
$$

where $\rho=\min _{\mu_{j} \neq \mu_{i}}\left|\mu_{j}-\mu_{i}\right|$ is a separation constant of $\mu_{i}$ and $\mathrm{C}_{4}$ is a positive constant independent of $h$.

Proof. From (3.2) in [23], (2.25) in [11], and (27), we have

$$
\begin{aligned}
& \operatorname{dist}\left(u_{j}^{h}, M_{h}\left(\lambda_{i}\right)\right) \\
& \leq \frac{16}{\rho}\left|\frac{1}{\lambda_{0}}-\frac{1}{\lambda_{j, h}}\right| \operatorname{dist}\left(u_{0}, M_{h}\left(\lambda_{i}\right)\right) \\
& \leq C_{4}\left(\left|\lambda_{0}-\lambda_{i}\right|+C\left(\lambda_{i}\right) \delta_{h}^{2}\left(\lambda_{i}\right)\right) \operatorname{dist}\left(u_{0}, M_{h}\left(\lambda_{i}\right)\right) .
\end{aligned}
$$

The proof is completed.

\section{One Correction Step Based on the Shifted Inverse Iteration}

We first generate a coarse mesh $\mathscr{T}_{H}$ with the mesh size $H$. The coarse linear finite element space $V_{H}$ is defined on $\mathscr{T}_{H}$. Then we define a sequence of triangulations $\mathscr{T}_{h_{k}}$ of $\Omega$ determined as follows. Set $h_{1}=H$ and let $\mathscr{T}_{h_{k}}$ be obtained from $\mathscr{T}_{h_{k-1}}$ via regular refinement. Then we construct the linear finite element spaces such that

$$
\begin{aligned}
V_{H} & =V_{h_{1}} \subset V_{h_{2}} \subset \cdots \subset V_{h_{n}}, \\
\delta_{h_{k+1}}\left(\lambda_{i}\right) & =\delta_{h_{k}}^{1+\gamma}\left(\lambda_{i}\right),
\end{aligned}
$$

where $\gamma \in(0,2]$.

Assume that we have the eigenpair approximations $\left(\tilde{\lambda}_{j, h_{k}}, \tilde{u}_{j, h_{k}}\right) \in \mathscr{R} \times V_{H, h_{k}}(j=i, \ldots, i+q-1)$, where the eigenvalues $\left\{\tilde{\lambda}_{j, h_{k}}\right\}_{j=i}^{i+q-1}$ are the approximations of the eigenvalue $\lambda_{i}$ of (2) and $V_{H, h_{k}}$ is an extended space (see Algorithm 4). Now we give the following one correction step to improve the accuracy of the current eigenpair approximations $\left\{\widetilde{\lambda}_{j, h_{k}}, \tilde{u}_{j, h_{k}}\right\}_{j=i}^{i+q-1}$.

Algorithm 4 (one correction step).

Step 1. For $j=i, \ldots, i+q-1$, do the following.

Find $\bar{u}_{j, h_{k+1}} \in V_{h_{k+1}}$ such that

$a\left(\bar{u}_{j, h_{k+1}}, v\right)-\tilde{\lambda}_{i, h_{k}} b\left(\bar{u}_{j, h_{k+1}}, v\right)=b\left(\tilde{u}_{j, h_{k}}, v\right)$,

and set $\widehat{u}_{j, h_{k+1}}=\bar{u}_{j, h_{k+1}} /\left\|\bar{u}_{j, h_{k+1}}\right\|_{a}$. 
Step 2. Define a new finite element space $V_{H, h_{k+1}}=V_{H} \oplus$ $\operatorname{span}\left\{\widehat{u}_{i, h_{k+1}}, \ldots, \widehat{u}_{i+q-1, h_{k+1}}\right\}$ and solve the following Steklov eigenvalue problem. and

Find $\left(\widetilde{\lambda}_{j, h_{k+1}}, \widetilde{u}_{j, h_{k+1}}\right) \in \mathscr{R} \times V_{H, h_{k+1}}$ such that $\left\|\tilde{u}_{j, h_{k+1}}\right\|_{a}=1$

$$
a\left(\tilde{u}_{j, h_{k+1}}, v\right)=\tilde{\lambda}_{j, h_{k+1}} b\left(\tilde{u}_{j, h_{k+1}}, v\right), \quad \forall v \in V_{H, h_{k+1}} .
$$

Output the eigenvalues $\left\{\tilde{\lambda}_{j, h_{k+1}}\right\}_{j=i}^{i+q-1}$ and the corresponding orthonormal eigenfunctions $\left\{\tilde{u}_{j, h_{k+1}}\right\}_{j=i}^{i+q-1}$ with respect to $a(\cdot, \cdot)$.

We adopt the notations in [15-17] to simplify and summarize Algorithm 4 as

$$
\begin{aligned}
& \left\{\widetilde{\lambda}_{j, h_{k+1}}, \tilde{u}_{j, h_{k+1}}\right\}_{j=i}^{i+q-1} \\
& =\text { Correction }\left(V_{H},\left\{\widetilde{\lambda}_{j, h_{k}}, \widetilde{u}_{j, h_{k}}\right\}_{j=i}^{i+q-1}, V_{h_{k+1}}\right) .
\end{aligned}
$$

Theorem 5. Suppose that $H$ is properly small. Assume the eigenpairs $\left\{\tilde{\lambda}_{j, h_{k}}, \widetilde{u}_{j, h_{k}}\right\}_{j=i}^{i+q-1}$ in Algorithm 4 have the following error estimates:

$$
\begin{aligned}
\Theta\left(M\left(\lambda_{i}\right), M_{H, h_{k}}\left(\lambda_{i}\right)\right) & \leq C_{0} \delta_{h_{k}}\left(\lambda_{i}\right), \\
\Phi\left(M\left(\lambda_{i}\right), M_{H, h_{k}}\left(\lambda_{i}\right)\right) & \leq C_{0} \eta_{a}(H) \delta_{h_{k}}\left(\lambda_{i}\right), \\
\left|\lambda_{i}-\tilde{\lambda}_{j, h_{k}}\right| & \leq C_{0} \delta_{h_{k}}^{2}\left(\lambda_{i}\right) .
\end{aligned}
$$

Then, after one correction step, the resulting eigenpair approximations $\left\{\widetilde{\lambda}_{j, h_{k+1}}, \widetilde{u}_{j, h_{k+1}}\right\}_{j=i}^{i+q-1}$ have the following error estimates:

$$
\begin{aligned}
\Theta\left(M\left(\lambda_{i}\right), M_{H, h_{k+1}}\left(\lambda_{i}\right)\right) & \leq C_{0} \delta_{h_{k+1}}\left(\lambda_{i}\right), \\
\Phi\left(M\left(\lambda_{i}\right), M_{H, h_{k+1}}\left(\lambda_{i}\right)\right) & \leq C_{0} \eta_{a}(H) \delta_{h_{k+1}}\left(\lambda_{i}\right), \\
\left|\lambda_{i}-\tilde{\lambda}_{j, h_{k+1}}\right| & \leq C_{0} \delta_{h_{k+1}}^{2}\left(\lambda_{i}\right),
\end{aligned}
$$

where

$$
C_{0}=\max \left\{2 q^{3 / 2} C_{5} C^{2}\left(\lambda_{i}\right), 4 q^{3} C_{5}^{2} C^{3}\left(\lambda_{i}\right), C\left(\lambda_{i}\right)\right\}
$$

is a positive constant dependent on $\lambda_{i}$ but independent of $k$ and $h$, and $C_{5}=1$ when $\lambda_{i}$ is a simple eigenvalue, and when $\lambda_{i}$ is a multiple eigenvalue $C_{5}$ is a positive constant independent of $k$ and $h$ (see Lemma 6 at the back).

Proof. From (35), we know there exists a basis $\left\{u_{j}\right\}_{j=i}^{i+q-1}$ of $M\left(\lambda_{i}\right)$ such that

$$
\begin{aligned}
\left\|u_{j}-\tilde{u}_{j, h_{k}}\right\|_{a} & \leq C_{0} \delta_{h_{k}}\left(\lambda_{i}\right), \\
\left\|u_{j}-\tilde{u}_{j, h_{k}}\right\|_{b} & \leq C_{0} \eta_{a}(H) \delta_{h_{k}}\left(\lambda_{i}\right), \\
\left|\lambda_{i}-\tilde{\lambda}_{i, h_{k}}\right| & \leq C_{0} \delta_{h_{k}}^{2}\left(\lambda_{i}\right) .
\end{aligned}
$$

By (15), we know that (32) is equivalent to the following:

$$
\begin{array}{r}
a\left(\bar{u}_{j, h_{k+1}}, v\right)-\tilde{\lambda}_{i, h_{k}} a\left(T_{h_{k+1}} \bar{u}_{j, h_{k+1}}, v\right) \\
\quad=a\left(T_{h_{k+1}} \tilde{u}_{j, h_{k}}, v\right), \quad \forall v \in V_{h_{k+1}},
\end{array}
$$

and $\widehat{u}_{j, h_{k+1}}=\bar{u}_{j, h_{k+1}} /\left\|\bar{u}_{j, h_{k+1}}\right\|_{a}$; that is,

$$
\begin{aligned}
\left(\frac{1}{\widetilde{\lambda}_{i, h_{k}}}-T_{h_{k+1}}\right) \bar{u}_{j, h_{k+1}} & =\tilde{\lambda}_{i, h_{k}}^{-1} T_{h_{k+1}} \tilde{u}_{j, h_{k}}, \\
\widehat{u}_{j, h_{k+1}} & =\frac{\bar{u}_{j, h_{k+1}}}{\left\|\bar{u}_{j, h_{k+1}}\right\|_{a}} .
\end{aligned}
$$

Select $u_{j, h_{k+1}}^{0}=\tilde{\lambda}_{i, h_{k}} T_{h_{k+1}} \tilde{u}_{j, h_{k}} /\left\|\tilde{\lambda}_{i, h_{k}} T_{h_{k+1}} \tilde{u}_{j, h_{k}}\right\|_{a} ;$ then $\left\|u_{j, h_{k+1}}^{0}\right\|_{a}=1$ and $u_{j, h_{k+1}}^{0} \in V_{h_{k+1}}$. Note that $\widetilde{\lambda}_{i, h_{k}}^{-1} T_{h_{k+1}} \tilde{u}_{j, h_{k}}=$ $\left\|\tilde{\lambda}_{i, h_{k}}^{-1} T_{h_{k+1}} \tilde{u}_{j, h_{k}}\right\|_{a} u_{j, h_{k+1}}^{0}$ differs from $u_{j, h_{k+1}}^{0}$ by only a constant; then (32) is equivalent to

$$
\begin{aligned}
\left(\frac{1}{\widetilde{\lambda}_{i, h_{k}}}-T_{h_{k+1}}\right) \bar{u}_{j, h_{k+1}} & =u_{j, h_{k+1}}^{0}, \\
\widehat{u}_{j, h_{k+1}} & =\frac{\bar{u}_{j, h_{k+1}}}{\left\|\bar{u}_{j, h_{k+1}}\right\|_{a}} .
\end{aligned}
$$

Let $\lambda_{j, h_{k+1}}$ be the $j$ th eigenvalue of (7) with $h=h_{k+1}$; then thanks to Lemma 3 we have

$$
\begin{aligned}
& \operatorname{dist}\left(\widehat{u}_{j, h_{k+1}}, M_{h_{k+1}}\left(\lambda_{i}\right)\right) \leq \frac{16}{\rho}\left|\frac{1}{\widetilde{\lambda}_{i, h_{k}}}-\frac{1}{\lambda_{j, h_{k+1}}}\right| \\
& \cdot \operatorname{dist}\left(u_{j, h_{k+1}}^{0}, M_{h_{k+1}}\left(\lambda_{i}\right)\right) \\
& \leq C_{4}\left\{\left|\widetilde{\lambda}_{i, h_{k}}-\lambda_{i}\right|+C\left(\lambda_{i}\right) \delta_{h_{k+1}}^{2}\left(\lambda_{i}\right)\right\} \\
& \cdot \operatorname{dist}\left(u_{j, h_{k+1}}^{0}, M_{h_{k+1}}\left(\lambda_{i}\right)\right) \\
& \leq\left\{C_{0} C_{4} \delta_{h_{k}}^{2}\left(\lambda_{i}\right)+C_{4} C\left(\lambda_{i}\right) \delta_{h_{k+1}}^{2}\left(\lambda_{i}\right)\right\} \\
& \quad \cdot \operatorname{dist}\left(u_{j, h_{k+1}}^{0}, M_{h_{k+1}}\left(\lambda_{i}\right)\right) .
\end{aligned}
$$

Then, using the triangle inequality and Lemma 2, we get

$$
\begin{aligned}
& \operatorname{dist}\left(u_{j, h_{k+1}}^{0}, M_{h_{k+1}}\left(\lambda_{i}\right)\right) \\
& \quad \leq \operatorname{dist}\left(u_{j, h_{k+1}}^{0}, M\left(\lambda_{i}\right)\right)+C\left(\lambda_{i}\right) \delta_{h_{k+1}}\left(\lambda_{i}\right) .
\end{aligned}
$$

Combining (46) and (47), we obtain

$$
\begin{aligned}
& \operatorname{dist}\left(\widehat{u}_{j, h_{k+1}}, M_{h_{k+1}}\left(\lambda_{i}\right)\right) \\
& \leq\left\{C_{0} C_{4} \delta_{h_{k}}^{2}\left(\lambda_{i}\right)+C_{4} C\left(\lambda_{i}\right) \delta_{h_{k+1}}^{2}\left(\lambda_{i}\right)\right\} \\
& \cdot \operatorname{dist}\left(u_{j, h_{k+1}}^{0}, M\left(\lambda_{i}\right)\right)+C_{4} C\left(\lambda_{i}\right) \\
& \quad \cdot\left\{C_{0} \delta_{h_{k}}^{2}\left(\lambda_{i}\right)+C\left(\lambda_{i}\right) \delta_{h_{k+1}}^{2}\left(\lambda_{i}\right)\right\} \delta_{h_{k+1}}\left(\lambda_{i}\right) .
\end{aligned}
$$

Let the eigenvectors $\left\{u_{j, h_{k+1}}\right\}_{j=i}^{i+q-1}$ be an orthonormal basis of $M_{h_{k+1}}\left(\lambda_{i}\right)$ with respect to $a(\cdot, \cdot)$. Denote

$$
\widehat{u}_{j}^{*}=\sum_{m=i}^{i+q-1} a\left(\widehat{u}_{j, h_{k+1}}, u_{m, h_{k+1}}\right) u_{m, h_{k+1}} ;
$$


then

$$
\left\|\widehat{u}_{j, h_{k+1}}-\widehat{u}_{j}^{*}\right\|_{a}=\operatorname{dist}\left(\widehat{u}_{j, h_{k+1}}, M_{h_{k+1}}\left(\lambda_{i}\right)\right) .
$$

By (25), there exists $\left\{u_{j}^{\prime}\right\}_{j=i}^{i+q-1} \subset M\left(\lambda_{i}\right)$ satisfying

$$
\left\|u_{j, h_{k+1}}-u_{j}^{\prime}\right\|_{a} \leq C\left(\lambda_{i}\right) \delta_{h_{k+1}}\left(\lambda_{i}\right) .
$$

Let

$$
\widehat{u}_{j}=\sum_{m=i}^{i+q-1} a\left(\widehat{u}_{j, h_{k+1}}, u_{m, h_{k+1}}\right) u_{m}^{\prime} .
$$

Noting that $\left\{\tilde{u}_{j, h_{k}}\right\}_{j=i}^{i+q-1}$ is linearly independent, it can be deduced from (45), (49), and (52) that $\left\{\widehat{u}_{j}\right\}_{j=i}^{i+q-1}$ is linearly independent.

Using (49), (51), and (52), we deduce that

$$
\begin{aligned}
\left\|\widehat{u}_{j}-\widehat{u}_{j}^{*}\right\|_{a} & =\left\|\sum_{m=i}^{i+q-1} a\left(\widehat{u}_{j, h_{k+1}}, u_{m, h_{k+1}}\right)\left(u_{m}^{\prime}-u_{m, h_{k+1}}\right)\right\|_{a} \\
& \leq\left(\sum_{m=i}^{i+q-1}\left\|u_{m}^{\prime}-u_{m, h_{k+1}}\right\|_{a}^{2}\right)^{1 / 2} \\
& \leq\left\{\sum_{m=i}^{i+q-1} C^{2}\left(\lambda_{i}\right) \delta_{h_{k+1}}^{2}\left(\lambda_{i}\right)\right\}^{1 / 2} \\
& \leq q^{1 / 2} C\left(\lambda_{i}\right) \delta_{h_{k+1}}\left(\lambda_{i}\right) .
\end{aligned}
$$

Thus, using the triangle inequality, we have

$$
\begin{aligned}
\left\|\widehat{u}_{j, h_{k+1}}-\widehat{u}_{j}\right\|_{a} \leq & \operatorname{dist}\left(\widehat{u}_{j, h_{k+1}}, M_{h_{k+1}}\left(\lambda_{i}\right)\right) \\
& +q^{1 / 2} C\left(\lambda_{i}\right) \delta_{h_{k+1}}\left(\lambda_{i}\right),
\end{aligned}
$$

which together with (48) yields

$$
\begin{aligned}
\left\|\widehat{u}_{j, h_{k+1}}-\widehat{u}_{j}\right\|_{a} \leq\left\{C_{4} C_{0} \delta_{h_{k}}^{2}\left(\lambda_{i}\right)+C_{4} C\left(\lambda_{i}\right) \delta_{h_{k+1}}^{2}\left(\lambda_{i}\right)\right\} \\
\cdot \operatorname{dist}\left(u_{j, h_{k+1}}^{0}, M\left(\lambda_{i}\right)\right)+C\left(\lambda_{i}\right) \\
\quad \cdot\left\{C_{4} C_{0} \delta_{h_{k}}^{2}\left(\lambda_{i}\right)+C_{4} C\left(\lambda_{i}\right) \delta_{h_{k+1}}^{2}\left(\lambda_{i}\right)+q^{1 / 2}\right\} \\
\cdot \delta_{h_{k}+1}\left(\lambda_{i}\right) .
\end{aligned}
$$
have

Noting that $\left\|u_{j}\right\|_{a}=1$ and using Lemma 3.1 in [23], we

$$
\begin{aligned}
\operatorname{dist}\left(u_{j, h_{k+1}}^{0}, M\left(\lambda_{i}\right)\right) & \leq\left\|u_{j, h_{k+1}}^{0}-u_{j}\right\|_{a} \\
& \leq 2\left\|\tilde{\lambda}_{i, h_{k}} T_{h_{k+1}} \tilde{u}_{j, h_{k}}-u_{j}\right\|_{a} .
\end{aligned}
$$

Since $u_{j}=\lambda_{j} T u_{j}$ and $\left\|\tilde{u}_{j, h_{k}}\right\|_{a}=1$, from (17), (41), and (42), we have

$$
\begin{aligned}
& \left\|\widetilde{\lambda}_{i, h_{k}} T_{h_{k+1}} \widetilde{u}_{j, h_{k}}-u_{j}\right\|_{a}=\| \tilde{\lambda}_{i, h_{k}} T_{h_{k+1}} \tilde{u}_{j, h_{k}}-\lambda_{j} T_{h_{k+1}} \widetilde{u}_{j, h_{k}} \\
& \quad+\lambda_{j} T_{h_{k+1}} \widetilde{u}_{j, h_{k}}-\lambda_{j} T_{h_{k+1}} u_{j}+\lambda_{j} T_{h_{k+1}} u_{j}-\lambda_{j} T u_{j} \|_{a} \\
& \quad \leq\left|\widetilde{\lambda}_{i, h_{k}}-\lambda_{j}\right|\left\|T_{h_{k+1}} \widetilde{u}_{j, h_{k}}\right\|_{a} \\
& \quad+\lambda_{j}\left\|T_{h_{k+1}}\left(\widetilde{u}_{j, h_{k}}-u_{j}\right)\right\|_{a}+\left\|\lambda_{j}\left(T_{h_{k+1}}-T\right) u_{j}\right\|_{a} \\
& \quad \leq C_{0} C_{3} \delta_{h_{k}}^{2}\left(\lambda_{i}\right)+C_{3} \lambda_{i}\left\|\tilde{u}_{j, h_{k}}-u_{j}\right\|_{b}+\delta_{h_{k+1}}\left(\lambda_{i}\right) \\
& \quad \leq C_{0} C_{3} \delta_{h_{k}}^{2}\left(\lambda_{i}\right)+C_{0} C_{3} \lambda_{i} \eta_{a}(H) \delta_{h_{k}}\left(\lambda_{i}\right) \\
& \quad+\delta_{h_{k+1}}\left(\lambda_{i}\right) .
\end{aligned}
$$

From (56) and (57), we get

$$
\begin{aligned}
\operatorname{dist}\left(u_{j, h_{k+1}}^{0}, M\left(\lambda_{i}\right)\right) \leq & 2 C_{0} C_{3} \delta_{h_{k}}^{2}\left(\lambda_{i}\right) \\
& +2 C_{0} C_{3} \lambda_{i} \eta_{a}(H) \delta_{h_{k}}\left(\lambda_{i}\right) \\
& +2 \delta_{h_{k+1}}\left(\lambda_{i}\right) .
\end{aligned}
$$

Combining (55) and (58), reminding that $\delta_{h_{k+1}}=\delta_{h_{k}}^{1+\gamma}, \gamma \in$ $(0,2]$, we can obtain

$$
\begin{aligned}
& \left\|\widehat{u}_{j, h_{k+1}}-\widehat{u}_{j}\right\|_{a} \leq\left\{C_{0} C_{4} \delta_{h_{k}}^{2}\left(\lambda_{i}\right)+C_{4} C\left(\lambda_{i}\right) \delta_{h_{k+1}}^{2}\left(\lambda_{i}\right)\right\} \\
& \quad \cdot\left\{2 C_{0} C_{3} \delta_{h_{k}}^{2}\left(\lambda_{i}\right)\right. \\
& \left.+2 C_{0} C_{3} \lambda_{i} \eta_{a}(H) \delta_{h_{k}}\left(\lambda_{i}\right)+2 \delta_{h_{k+1}}\left(\lambda_{i}\right)\right\} \\
& +\left\{C_{0} C_{4} C\left(\lambda_{i}\right) \delta_{h_{k}}^{2}\left(\lambda_{i}\right)+C_{4} C^{2}\left(\lambda_{i}\right) \delta_{h_{k+1}}^{2}\left(\lambda_{i}\right)\right. \\
& \left.+q^{1 / 2} C\left(\lambda_{i}\right)\right\} \delta_{h_{k}+1}\left(\lambda_{i}\right)=2 C_{0}^{2} C_{3} C_{4} \delta_{h_{k}}^{1+\gamma}\left(\lambda_{i}\right) \\
& \cdot \delta_{h_{k}}^{3-\gamma}\left(\lambda_{i}\right)+2 C_{0}^{2} C_{3} C_{4} \lambda_{i} \eta_{a}(H) \delta_{h_{k}}^{1+\gamma}\left(\lambda_{i}\right) \delta_{h_{k}}^{2-\gamma}\left(\lambda_{i}\right) \\
& +2 C_{0} C_{4} \delta_{h_{k}}^{2}\left(\lambda_{i}\right) \delta_{h_{k+1}}\left(\lambda_{i}\right)+2 C_{0} C_{3} C_{4} C\left(\lambda_{i}\right) \\
& \cdot \delta_{h_{k}}^{2}\left(\lambda_{i}\right) \delta_{h_{k+1}}^{2}\left(\lambda_{i}\right)+2 C_{0} C_{3} C_{4} C\left(\lambda_{i}\right) \lambda_{i} \eta_{a}(H) \\
& \cdot \delta_{h_{k}}\left(\lambda_{i}\right) \delta_{h_{k+1}}^{2}\left(\lambda_{i}\right)+2 C_{4} C\left(\lambda_{i}\right) \delta_{h_{k+1}}^{3}\left(\lambda_{i}\right) \\
& +\left\{C_{0} C_{4} C\left(\lambda_{i}\right) \delta_{h_{k}}^{2}\left(\lambda_{i}\right)+C_{4} C^{2}\left(\lambda_{i}\right) \delta_{h_{k+1}}^{2}\left(\lambda_{i}\right)\right. \\
& \left.+q^{1 / 2} C\left(\lambda_{i}\right)\right\} \delta_{h_{k}+1}\left(\lambda_{i}\right)=\left\{2 C_{0}^{2} C_{3} C_{4} \delta_{h_{k}}^{3-\gamma}\left(\lambda_{i}\right)\right. \\
& +2 C_{0}^{2} C_{3} C_{4} \lambda_{i} \eta_{a}(H) \delta_{h_{k}}^{2-\gamma}\left(\lambda_{i}\right)+2 C_{0} C_{4} \delta_{h_{k}}^{2}\left(\lambda_{i}\right) \\
& +2 C_{0} C_{3} C_{4} C\left(\lambda_{i}\right) \delta_{h_{k}}^{2}\left(\lambda_{i}\right) \delta_{h_{k+1}}\left(\lambda_{i}\right) \\
& +2 C_{0} C_{3} C_{4} C\left(\lambda_{i}\right) \lambda_{i} \eta_{a}(H) \delta_{h_{k}}\left(\lambda_{i}\right) \delta_{h_{k+1}}\left(\lambda_{i}\right) \\
& +2 C_{4} C\left(\lambda_{i}\right) \delta_{h_{k+1}}^{2}\left(\lambda_{i}\right)+C_{0} C_{4} C\left(\lambda_{i}\right) \delta_{h_{k}}^{2}\left(\lambda_{i}\right) \\
& \left.+C_{4} C^{2}\left(\lambda_{i}\right) \delta_{h_{k+1}}^{2}\left(\lambda_{i}\right)+q^{1 / 2} C\left(\lambda_{i}\right)\right\} \delta_{h_{k}+1}\left(\lambda_{i}\right)
\end{aligned}
$$




$$
\begin{aligned}
& \leq\left\{2 C_{0}^{2} C_{3} C_{4} \delta_{H}\left(\lambda_{i}\right)+2 C_{0}^{2} C_{3} C_{4} \lambda_{i} \eta_{a}(H)\right. \\
& +2 C_{0} C_{4} \delta_{H}^{2}\left(\lambda_{i}\right)+2 C_{0} C_{3} C_{4} C\left(\lambda_{i}\right) \delta_{H}^{3}\left(\lambda_{i}\right) \\
& +2 C_{0} C_{3} C_{4} C\left(\lambda_{i}\right) \lambda_{i} \eta_{a}(H) \delta_{H}^{2}\left(\lambda_{i}\right) \\
& +2 C_{4} C\left(\lambda_{i}\right) \delta_{H}^{2}\left(\lambda_{i}\right)+C_{0} C_{4} C\left(\lambda_{i}\right) \delta_{H}^{2}\left(\lambda_{i}\right) \\
& \left.+C_{4} C^{2}\left(\lambda_{i}\right) \delta_{H}^{2}\left(\lambda_{i}\right)+q^{1 / 2} C\left(\lambda_{i}\right)\right\} \delta_{h_{k+1}}\left(\lambda_{i}\right) .
\end{aligned}
$$

When $H$ is properly small, we have

$$
\begin{aligned}
2 C_{0}^{2} C_{3} C_{4} \delta_{H}\left(\lambda_{i}\right)+2 C_{0}^{2} C_{3} C_{4} \lambda_{i} \eta_{a}(H) \\
+2 C_{0} C_{4} \delta_{H}^{2}\left(\lambda_{i}\right)+2 C_{0} C_{3} C_{4} C\left(\lambda_{i}\right) \delta_{H}^{3}\left(\lambda_{i}\right) \\
+2 C_{0} C_{3} C_{4} C\left(\lambda_{i}\right) \lambda_{i} \eta_{a}(H) \delta_{H}^{2}\left(\lambda_{i}\right) \\
+2 C_{4} C\left(\lambda_{i}\right) \delta_{H}^{2}\left(\lambda_{i}\right)+C_{0} C_{4} C\left(\lambda_{i}\right) \delta_{H}^{2}\left(\lambda_{i}\right) \\
+C_{4} C^{2}\left(\lambda_{i}\right) \delta_{H}^{2}\left(\lambda_{i}\right) \leq q^{1 / 2} C\left(\lambda_{i}\right) ;
\end{aligned}
$$

then we obtain the following error estimate:

$$
\left\|\widehat{u}_{j, h_{k+1}}-\widehat{u}_{j}\right\|_{a} \leq 2 q^{1 / 2} C\left(\lambda_{i}\right) \delta_{h_{k+1}}\left(\lambda_{i}\right)
$$

Now we come to estimate the error for the eigenpairs $\left\{\widetilde{\lambda}_{j, h_{k+1}}, \tilde{u}_{j, h_{k+1}}\right\}_{j=i}^{i+q-1}$. Based on the error estimate theory of eigenvalue problem by finite element methods (see, e.g., [4, 26]), from Lemmas 2 and 6 at the back, (61), and the definition of the space $V_{H, h_{k+1}}$, we have the following estimates:

$$
\begin{aligned}
& \Theta\left(M\left(\lambda_{i}\right), M_{H, h_{k+1}}\left(\lambda_{i}\right)\right) \leq C\left(\lambda_{i}\right) \\
& \cdot \sup _{w \in M\left(\lambda_{i}\right)} \inf _{v_{H, h_{k+1}} \in V_{H, h_{k+1}}}\left\|w-v_{H, h_{k+1}}\right\|_{a} \leq C\left(\lambda_{i}\right) \\
& \cdot \sup _{\beta_{j}}\left\{\left\|\sum_{j=i}^{i+q-1} \beta_{j} \widehat{u}_{j}-\sum_{m=i}^{i+q-1} \beta_{m} \widehat{u}_{m, h_{k+1}}\right\|_{a}\right\} \leq C\left(\lambda_{i}\right) \\
& \cdot \sup _{\beta_{j}} \sum_{j=i}^{i+q-1}\left|\beta_{j}\right|\left\|\widehat{u}_{j}-\widehat{u}_{j, h_{k+1}}\right\|_{a} \leq q C_{5} C\left(\lambda_{i}\right) \\
& \cdot \max \left\{\left\|\widehat{u}_{i, h_{k+1}}-\widehat{u}_{i}\right\|_{a}, \ldots,\left\|\widehat{u}_{i+q-1, h_{k+1}}-\widehat{u}_{i+q-1}\right\|_{a}\right\} \\
& \leq 2 q^{3 / 2} C_{5} C^{2}\left(\lambda_{i}\right) \delta_{h_{k+1}}\left(\lambda_{i}\right), \\
& \Phi\left(M\left(\lambda_{i}\right), M_{H, h_{k+1}}\left(\lambda_{i}\right)\right) \leq C\left(\lambda_{i}\right) \\
& \cdot \sup _{w \in M\left(\lambda_{i}\right)} \inf _{v_{H, h_{k+1}} \in V_{H, h_{k+1}}}\left\|w-v_{H, h_{k+1}}\right\|_{b} \leq C\left(\lambda_{i}\right) \\
& \cdot \widetilde{\eta}_{a}(H) \sup _{w \in M\left(\lambda_{i}\right)} \inf _{v_{H, h_{k+1}} \in V_{H, h_{k+1}}}\left\|w-v_{H, h_{k+1}}\right\|_{a} \\
& \leq 2 q^{3 / 2} C_{5} C^{2}\left(\lambda_{i}\right) \widetilde{\eta}_{a}(H) \delta_{h_{k+1}}\left(\lambda_{i}\right),
\end{aligned}
$$

$$
\left|\beta_{j}\right| \leq C_{5}, \quad \text { for } j=i, \ldots, i+q-1,
$$

where $C_{5}$ is a constant independent of $k$ and $h$.

Proof. From the proof of Theorem 5 we know that $\left\{\widehat{u}_{j}\right\}_{j=i}^{i+q-1}$ is linearly independent, so it can be taken as a basis of $M\left(\lambda_{i}\right)$. For any $w \in M\left(\lambda_{i}\right), w=\sum_{j=i}^{i+q-1} \beta_{j} \widehat{u}_{j}$, we have

$$
\begin{aligned}
1 & =\|w\|_{a}^{2}=\left\|\sum_{m=i}^{i+q-1} \beta_{m} \widehat{u}_{m}\right\|_{a}^{2} \\
& =\sum_{m=i}^{i+q-1} \beta_{m}^{2}\left\|\widehat{u}_{m}\right\|_{a}^{2}+\sum_{m, j=i, m \neq j}^{i+q-1} \beta_{m} \beta_{j} a\left(\widehat{u}_{m}, \widehat{u}_{j}\right) .
\end{aligned}
$$

Noticing that the norm of self-adjoint operator is equal to its spectral radius, then

$$
\begin{aligned}
\left\|\left(\mu_{0}-T_{h_{k+1}}\right)^{-1}\right\|_{a} & =\left(\min _{j}\left|\mu_{0}-\mu_{j, h_{k+1}}\right|\right)^{-1} \\
& \equiv\left(\left|\mu_{0}-\mu_{j_{0}, h_{k+1}}\right|\right)^{-1}
\end{aligned}
$$

where $\mu_{0}=1 / \lambda_{0}=1 / \widetilde{\lambda}_{i, h_{k}}$. 
Mathematical Problems in Engineering

7

For $u_{j}$ in (40)-(41), by calculation, we deduce

$$
\begin{aligned}
& \left\|\left(\mu_{0}-T_{h_{k+1}}\right)^{-1} \tilde{\lambda}_{i, h_{k}} T_{h_{k+1}} \tilde{u}_{j, h_{k}}-\left(\mu_{0}-\mu_{j_{0}, h_{k+1}}\right)^{-1} u_{j}\right\|_{a} \\
& =\|\left(\mu_{0}-T_{h_{k+1}}\right)^{-1}\left(\tilde{\lambda}_{i, h_{k}} T_{h_{k+1}} \tilde{u}_{j, h_{k}}-u_{j}\right)+\left(\mu_{0}\right. \\
& \left.-T_{h_{k+1}}\right)^{-1} u_{j}-\left(\mu_{0}-\mu_{j_{0}, h_{k+1}}\right)^{-1} u_{j}\left\|_{a}=\right\|\left(\mu_{0}\right. \\
& \left.-T_{h_{k+1}}\right)^{-1}\left(\tilde{\lambda}_{i, h_{k}} T_{h_{k+1}} \tilde{u}_{j, h_{k}}-u_{j}\right)+\left(\mu_{0}-T_{h_{k+1}}\right)^{-1} \\
& \left\{u_{j}-\sum_{m=i}^{i+q-1} a\left(u_{j}, u_{m, h_{k+1}}\right) u_{m, h_{k+1}}\right\}+\left(\mu_{0}\right. \\
& \left.-T_{h_{k+1}}\right)^{-1}\left\{\sum_{m=i}^{i+q-1} a\left(u_{j}, u_{m, h_{k+1}}\right) u_{m, h_{k+1}}\right\}-\left(\mu_{0}\right. \\
& \left.-\mu_{j_{0}, h_{k+1}}\right)^{-1}\left\{u_{j}-\sum_{m=i}^{i+q-1} a\left(u_{j}, u_{m, h_{k+1}}\right) u_{m, h_{k+1}}\right\} \\
& -\left(\mu_{0}-\mu_{j_{0}, h_{k+1}}\right)^{-1}\left\{\sum_{m=i}^{i+q-1} a\left(u_{j}, u_{m, h_{k+1}}\right) u_{m, h_{k+1}}\right\} \|_{a} \\
& \leq\left\|\left(\mu_{0}-T_{h_{k+1}}\right)^{-1}\left(\widetilde{\lambda}_{i, h_{k}} T_{h_{k+1}} \tilde{u}_{j, h_{k}}-u_{j}\right)\right\|_{a}+\|\left(\mu_{0}\right. \\
& \left.-T_{h_{k+1}}\right)^{-1} \|_{a} \delta_{h_{k+1}}\left(\lambda_{i}\right)+\left|\left(\mu_{0}-\mu_{j_{0}, h_{k+1}}\right)^{-1}\right| \delta_{h_{k+1}}\left(\lambda_{i}\right) \\
& +\|\left\{\sum_{m=i}^{i+q-1} a\left(u_{j}, u_{m, h_{k+1}}\right)\left(\mu_{0}-\mu_{m, h_{k+1}}\right)^{-1} u_{m, h_{k+1}}\right\} \\
& -\left\{\sum_{m=i}^{i+q-1} a\left(u_{j}, u_{m, h_{k+1}}\right)\left(\mu_{0}-\mu_{j_{0}, h_{k+1}}\right)^{-1} u_{m, h_{k+1}}\right\} \|_{a} \\
& \leq\left\|\left(\mu_{0}-T_{h_{k+1}}\right)^{-1}\left(\tilde{\lambda}_{i, h_{k}} T_{h_{k+1}} \tilde{u}_{j, h_{k}}-u_{j}\right)\right\|_{a}+\|\left(\mu_{0}\right. \\
& \left.-T_{h_{k+1}}\right)^{-1} \|_{a} \delta_{h_{k+1}}\left(\lambda_{i}\right)+\left|\left(\mu_{0}-\mu_{j_{0}, h_{k+1}}\right)^{-1}\right| \delta_{h_{k+1}}\left(\lambda_{i}\right) \\
& +\left|\left(\mu_{0}-\mu_{j_{0}, h_{k+1}}\right)^{-1}\right| \|\left\{\sum_{m=i}^{i+q-1} a\left(u_{j}, u_{m, h_{k+1}}\right)\right. \\
& \left.\left[\frac{\left(\mu_{0}-\mu_{m, h_{k+1}}\right)^{-1}}{\left(\mu_{0}-\mu_{j_{0}, h_{k+1}}\right)^{-1}}\right] u_{m, h_{k+1}}\right\} \|_{a} \text {, }
\end{aligned}
$$

where

$$
\begin{aligned}
& \left|\frac{\left(\mu_{0}-\mu_{m, h_{k+1}}\right)^{-1}}{\left(\mu_{0}-\mu_{j_{0}, h_{k+1}}\right)^{-1}}-1\right|=\left|\frac{\mu_{0}-\mu_{j_{0}, h_{k+1}}}{\mu_{0}-\mu_{m, h_{k+1}}}-1\right| \\
& =\left|\frac{\mu_{m, h_{k+1}}-\mu_{j_{0}, h_{k+1}}}{\mu_{0}-\mu_{m, h_{k+1}}}\right| .
\end{aligned}
$$

$$
\tilde{\lambda}_{j, h_{k}}-\lambda_{i} \geq C_{5}^{\prime} \delta_{h_{k}}^{2}\left(\lambda_{i}\right), \quad \text { for } j=i, \ldots, i+q-1 .
$$

Noting that $\mu_{0}=1 / \lambda_{0}=1 / \widetilde{\lambda}_{i, h_{k}}$, then, for $m=i, \ldots, i+q-1$, we have

$$
\begin{aligned}
& \left|\frac{\mu_{m, h_{k+1}}-\mu_{j_{0}, h_{k+1}}}{\mu_{0}-\mu_{m, h_{k+1}}}\right|=\left|\frac{\lambda_{0}\left(\lambda_{j_{0}, h_{k+1}}-\lambda_{m, h_{k+1}}\right)}{\lambda_{j_{0}, h_{k+1}}\left(\lambda_{m, h_{k+1}}-\lambda_{0}\right)}\right| \\
& \leq \frac{2 \lambda_{0} C\left(\lambda_{i}\right) \delta_{h_{k+1}}^{2}\left(\lambda_{i}\right)}{\lambda_{j_{0}, h_{k+1}}\left(C_{5}^{\prime}-C\left(\lambda_{i}\right) \delta_{h_{k}}^{2 \varepsilon}\left(\lambda_{i}\right)\right) \delta_{h_{k}}^{2}\left(\lambda_{i}\right)} \\
& =\frac{2 \lambda_{0} C\left(\lambda_{i}\right) \delta_{h_{k}}^{2+2 \varepsilon}\left(\lambda_{i}\right)}{\lambda_{j_{0}, h_{k+1}}\left(C_{5}^{\prime}-C\left(\lambda_{i}\right) \delta_{h_{k}}^{2 \varepsilon}\left(\lambda_{i}\right)\right) \delta_{h_{k}}^{2}\left(\lambda_{i}\right)} \\
& =2 \frac{\lambda_{0} C\left(\lambda_{i}\right) \delta_{h_{k}}^{2 \varepsilon}\left(\lambda_{i}\right)}{\lambda_{j_{0}, h_{k+1}}\left(C_{5}^{\prime}-C\left(\lambda_{i}\right) \delta_{h_{k}}^{2 \varepsilon}\left(\lambda_{i}\right)\right)}
\end{aligned}
$$

From (45), we have

$$
\begin{gathered}
\bar{u}_{j, h_{k}+1}=\left(\mu_{0}-T_{h_{k+1}}\right)^{-1} \frac{\tilde{\lambda}_{i, h_{k}} T_{h_{k+1}} \tilde{u}_{j, h_{k}}}{\left\|\tilde{\lambda}_{i, h_{k}} T_{h_{k+1}} \tilde{u}_{j, h_{k}}\right\|_{a}}, \\
\widehat{u}_{j, h_{k+1}}=\frac{\left(\mu_{0}-T_{h_{k+1}}\right)^{-1} \tilde{\lambda}_{i, h_{k}} T_{h_{k+1}} \tilde{u}_{j, h_{k}}}{\left\|\left(\mu_{0}-T_{h_{k+1}}\right)^{-1} \tilde{\lambda}_{i, h_{k}} T_{h_{k+1}} \tilde{u}_{j, h_{k}}\right\|_{a}} .
\end{gathered}
$$

Since $V_{H, h_{k}} \subset V_{h_{k}} \subset V_{h_{k+1}}$, we get

$$
\tilde{\lambda}_{j, h_{k}} \geq \lambda_{j, h_{k+1}}, \quad \text { for } j=i, \ldots, i+q-1 .
$$

Note that

$$
u_{j}=\frac{\left|\left(\mu_{0}-\mu_{j_{0}, h_{k+1}}\right)^{-1}\right| u_{j}}{\left\|\left(\mu_{0}-\mu_{j_{0}, h_{k+1}}\right)^{-1} u_{j}\right\|_{a}}=\frac{-\left(\mu_{0}-\mu_{j_{0}, h_{k+1}}\right)^{-1} u_{j}}{\left\|\left(\mu_{0}-\mu_{j_{0}, h_{k+1}}\right)^{-1} u_{j}\right\|_{a}}
$$

then, from Lemma 3.1 in [23], (57), and (68)-(73), we get

$$
\begin{gathered}
\left\|\widehat{u}_{j, h_{k+1}}+u_{j}\right\|_{a}=\| \frac{\left(\mu_{0}-T_{h_{k+1}}\right)^{-1} \widetilde{\lambda}_{i, h_{k}} T_{h_{k+1}} \widetilde{u}_{j, h_{k}}}{\left\|\left(\mu_{0}-T_{h_{k+1}}\right)^{-1} \tilde{\lambda}_{i, h_{k}} T_{h_{k+1}} \tilde{u}_{j, h_{k}}\right\|_{a}} \\
-\frac{\left(\mu_{0}-\mu_{j_{0}, h_{k+1}}\right)^{-1} u_{j}}{\left\|\left(\mu_{0}-\mu_{j_{0}, h_{k+1}}\right)^{-1} u_{j}\right\|_{a} \|_{a} \leq 2} \\
\cdot \frac{1}{\left\|\left(\mu_{0}-\mu_{j_{0}, h_{k+1}}\right)^{-1} u_{j}\right\|_{a}} \|\left(\mu_{0}-T_{h_{k+1}}\right)^{-1}
\end{gathered}
$$




$$
\begin{aligned}
& \cdot \tilde{\lambda}_{i, h_{k}} T_{h_{k+1}} \tilde{u}_{j, h_{k}}-\left(\mu_{0}-\mu_{j_{0}, h_{k+1}}\right)^{-1} u_{j} \|_{a} \leq 2 \mid \mu_{0} \\
& -\mu_{j_{0}, h_{k+1}} \mid\left\{\left\|\left(\mu_{0}-T_{h_{k}+1}\right)^{-1}\left(\tilde{\lambda}_{i, h_{k}} T_{h_{k+1}} \tilde{u}_{j, h_{k}}-u_{j}\right)\right\|_{a}\right. \\
& +\left\|\left(\mu_{0}-T_{h_{k+1}}\right)^{-1}\right\| \delta_{h_{k+1}}\left(\lambda_{i}\right)+\left|\left(\mu_{0}-\mu_{j_{0}, h_{k+1}}\right)^{-1}\right| \\
& \cdot \delta_{h_{k+1}}\left(\lambda_{i}\right)+\left|\left(\mu_{0}-\mu_{j_{0}, h_{k+1}}\right)^{-1}\right| \\
& \left.\cdot \frac{2 q \lambda_{0} C\left(\lambda_{i}\right) \delta_{h_{k}}^{2 \varepsilon}\left(\lambda_{i}\right)}{\lambda_{j_{0}, h_{k+1}}\left(C_{5}^{\prime}-C\left(\lambda_{i}\right) \delta_{h_{k}}^{2 \varepsilon}\left(\lambda_{i}\right)\right)}\right\} \\
& \leq 2\left\|\tilde{\lambda}_{i, h_{k}} T_{h_{k+1}} \tilde{u}_{j, h_{k}}-u_{j}\right\|_{a}+4 \delta_{h_{k+1}}\left(\lambda_{i}\right) \\
& +\frac{4 q \lambda_{0} C\left(\lambda_{i}\right) \delta_{h_{k}}^{2 \varepsilon}\left(\lambda_{i}\right)}{\lambda_{j_{0}, h_{k+1}}\left(C_{5}^{\prime}-C\left(\lambda_{i}\right) \delta_{h_{k}}^{2 \varepsilon}\left(\lambda_{i}\right)\right)} \leq 2 C_{0} C_{3} \delta_{h_{k}}^{2}\left(\lambda_{i}\right) \\
& +2 C_{0} C_{3} \lambda_{i} \eta_{a}(H) \delta_{h_{k}}\left(\lambda_{i}\right)+6 \delta_{h_{k+1}}\left(\lambda_{i}\right)+4 q \\
& \frac{\lambda_{0} C\left(\lambda_{i}\right) \delta_{h_{k}}^{2 \varepsilon}\left(\lambda_{i}\right)}{\lambda_{j_{0}, h_{k+1}}\left(C_{5}^{\prime}-C\left(\lambda_{i}\right) \delta_{h_{k}}^{2 \varepsilon}\left(\lambda_{i}\right)\right)} .
\end{aligned}
$$

For $i \leq m, j \leq i+q-1$, from (76), we have

$$
\begin{aligned}
& \left|a\left(\widehat{u}_{m, h_{k+1}}, \widehat{u}_{j, h_{k+1}}\right)\right|=\mid a\left(\widehat{u}_{m, h_{k+1}}+u_{m}, \widehat{u}_{j, h_{k+1}}\right) \\
& -a\left(u_{m}, \widehat{u}_{j, h_{k+1}}\right)|=| a\left(\widehat{u}_{m, h_{k+1}}+u_{m}, \widehat{u}_{j, h_{k+1}}\right) \\
& \quad-a\left(u_{m}, \widehat{u}_{j, h_{k+1}}+u_{j}\right)+a\left(u_{m}, u_{j}\right) \mid \leq \| \widehat{u}_{m, h_{k+1}} \\
& \quad+u_{m}\left\|_{a}+\right\| \widehat{u}_{j, h_{k+1}}+u_{j} \|_{a}+\left|a\left(u_{m}, u_{j}\right)\right| \\
& \quad \leq 4 C_{0} C_{3} \delta_{h_{k}}^{2}\left(\lambda_{i}\right)+4 C_{0} C_{3} \lambda_{i} \eta_{a}(H) \delta_{h_{k}}\left(\lambda_{i}\right) \\
& \quad+12 \delta_{h_{k+1}}\left(\lambda_{i}\right)+8 q \frac{\lambda_{0} C\left(\lambda_{i}\right) \delta_{h_{k}}^{2 \varepsilon}\left(\lambda_{i}\right)}{\lambda_{j_{0}, h_{k+1}}\left(C_{5}^{\prime}-C\left(\lambda_{i}\right) \delta_{h_{k}}^{2 \varepsilon}\left(\lambda_{i}\right)\right)} \\
& \quad+\left|a\left(u_{m}, u_{j}\right)\right| .
\end{aligned}
$$

By $a\left(\widetilde{u}_{m, h_{k}}, \widetilde{u}_{j, h_{k}}\right)=\delta_{m j}$, from (40), we get

$$
\begin{aligned}
& \left|a\left(u_{m}, u_{j}\right)\right|=\mid a\left(u_{m}-\tilde{u}_{m, h_{k}}, u_{j}\right) \\
& \quad+a\left(\tilde{u}_{m, h_{k}}, u_{j}-\tilde{u}_{j, h_{k}}\right)+a\left(\tilde{u}_{m, h_{k}}, \tilde{u}_{j, h_{k}}\right) \mid \\
& \quad \leq 2 C_{0} \delta_{h_{k}}\left(\lambda_{i}\right)+\delta_{m j} ;
\end{aligned}
$$

thus

$$
\begin{aligned}
& \left|a\left(\widehat{u}_{m, h_{k+1}}, \widehat{u}_{j, h_{k+1}}\right)\right| \\
& \leq 4 C_{0} C_{3} \delta_{h_{k}}^{2}\left(\lambda_{i}\right)+4 C_{0} C_{3} \lambda_{i} \eta_{a}(H) \delta_{h_{k}}\left(\lambda_{i}\right) \\
& \quad+12 \delta_{h_{k+1}}\left(\lambda_{i}\right) \\
& \quad+8 q \frac{\lambda_{0} C\left(\lambda_{i}\right) \delta_{h_{k}}^{2 \varepsilon}\left(\lambda_{i}\right)}{\lambda_{j_{0}, h_{k+1}}\left(C_{5}^{\prime}-C\left(\lambda_{i}\right) \delta_{h_{k}}^{2 \varepsilon}\left(\lambda_{i}\right)\right)} \\
& \quad+2 C_{0} \delta_{h_{k}}\left(\lambda_{i}\right)+\delta_{m j}
\end{aligned}
$$

then, from (61) and (79), we have

$$
\begin{aligned}
& \left|a\left(\widehat{u}_{m}, \widehat{u}_{j}\right)\right|=\mid a\left(\widehat{u}_{m}-\widehat{u}_{m, h_{k+1}}, \widehat{u}_{j}\right) \\
& \quad+a\left(\widehat{u}_{m, h_{k+1}}, \widehat{u}_{j}-\widehat{u}_{j, h_{k+1}}\right)+a\left(\widehat{u}_{m, h_{k+1}}, \widehat{u}_{j, h_{k+1}}\right) \mid \\
& \quad \leq 4 q^{1 / 2} C\left(\lambda_{i}\right) \delta_{h_{k+1}}\left(\lambda_{i}\right)+4 C_{0} C_{3} \delta_{h_{k}}^{2}\left(\lambda_{i}\right) \\
& \quad+4 C_{0} C_{3} \lambda_{i} \eta_{a}(H) \delta_{h_{k}}\left(\lambda_{i}\right)+12 \delta_{h_{k+1}}\left(\lambda_{i}\right)+8 q \\
& \quad \cdot \frac{\lambda_{0} C\left(\lambda_{i}\right) \delta_{h_{k}}^{2 \varepsilon}\left(\lambda_{i}\right)}{\lambda_{j_{0}, h_{k+1}}\left(C_{5}^{\prime}-C\left(\lambda_{i}\right) \delta_{h_{k}}^{2 \varepsilon}\left(\lambda_{i}\right)\right)}+2 C_{0} \delta_{h_{k}}\left(\lambda_{i}\right) \\
& +\delta_{m j} .
\end{aligned}
$$

Thus there exists $C_{5}^{\prime \prime}$ such that

$$
\left|a\left(\widehat{u}_{m}, \widehat{u}_{j}\right)\right| \leq C_{5}^{\prime \prime}\left\{\delta_{h_{k}}\left(\lambda_{i}\right)\right\}^{\min \{1,2 \varepsilon\}}, \quad m \neq j .
$$

Then, from (67), we have

$$
\begin{aligned}
\sum_{m=i}^{i+q-1} \beta_{m}^{2} & =1-\sum_{m, j=i, m \neq j}^{i+q-1} \beta_{m} \beta_{j} a\left(\widehat{u}_{m}, \widehat{u}_{j}\right) \\
& \leq 1+q \sum_{m=i}^{i+q-1} \beta_{m}^{2} C_{5}^{\prime \prime}\left\{\delta_{h_{k}}\left(\lambda_{i}\right)\right\}^{\min \{1,2 \varepsilon\}}
\end{aligned}
$$

thus, we get

$$
\sum_{m=i}^{i+q-1} \beta_{m}^{2} \leq \frac{1}{1-q C_{5}^{\prime \prime}\left\{\delta_{h_{k}}\left(\lambda_{i}\right)\right\}^{\min \{1,2 \varepsilon\}}}
$$

that is, $\left\{\beta_{m}\right\}_{m=i}^{i+q-1}$ are uniformly bounded from above with respect to $h_{k}$.

\section{Multilevel Correction Scheme for the Steklov Eigenvalue Problem}

Based on Algorithm 4, we introduce the following multilevel correction scheme.

Algorithm 7 (multilevel correction scheme).

Step 1. Construct a series of finite element spaces $V_{H}=$ $V_{h_{1}}, V_{h_{2}}, \ldots, V_{h_{n}}$ such that (31) holds. 
Step 2. Compute $q$ eigenpair approximations $\left\{\lambda_{j, h_{1}}, u_{j, h_{1}}\right\}_{j=i}^{i+q-1}$ for the eigenpairs $\left\{\lambda_{j}, u_{j}\right\}_{j=i}^{i+q-1}$ by solving the following Steklov eigenvalue problem. and

Find $\left(\lambda_{j, h_{1}}, u_{j, h_{1}}\right) \in \mathscr{R} \times V_{h_{1}}$ such that $a\left(u_{j, h_{1}}, u_{j, h_{1}}\right)=1$

$$
a\left(u_{j, h_{1}}, v\right)=\lambda_{j, h_{1}} b\left(u_{j, h_{1}}, v\right), \quad \forall v \in V_{h_{1}}
$$

Step 3. $\tilde{\lambda}_{j, h_{1}} \Leftarrow \lambda_{j, h_{1}}$ and $\tilde{u}_{j, h_{1}} \Leftarrow u_{j, h_{1}}$, for $j=i, \ldots, i+q-1$. For $k=1, \ldots, n-1$, do

$$
\begin{aligned}
& \left\{\widetilde{\lambda}_{j, h_{k+1}}, \widetilde{u}_{j, h_{k+1}}\right\}_{j=i}^{i+q-1} \\
& \quad=\text { Correction }\left(V_{H},\left\{\widetilde{\lambda}_{j, h_{k}}, \widetilde{u}_{j, h_{k}}\right\}_{j=i}^{i+q-1}, V_{h_{k+1}}\right) .
\end{aligned}
$$

Finally, we obtain $q$ eigenpair approximations $\left\{\tilde{\lambda}_{j, h_{n}}\right.$, $\left.\tilde{u}_{j, h_{n}}\right\}_{j=i}^{i+q-1} \in \mathscr{R} \times V_{H, h_{n}}$.

Theorem 8. After implementing Algorithm 7, if $H$ is small enough, the resultant eigenpair approximations $\left\{\tilde{\lambda}_{j, h_{n}}, \tilde{u}_{j, h_{n}}\right\}_{j=i}^{i+q-1}$ have the following error estimates:

$$
\begin{aligned}
\Theta\left(M\left(\lambda_{i}\right), M_{H, h_{n}}\left(\lambda_{i}\right)\right) & \leq C_{0} \delta_{h_{n}}\left(\lambda_{i}\right), \\
\Phi\left(M\left(\lambda_{i}\right), M_{H, h_{n}}\left(\lambda_{i}\right)\right) & \leq C_{0} \eta_{a}(H) \delta_{h_{n}}\left(\lambda_{i}\right), \\
\left|\lambda_{i}-\tilde{\lambda}_{j, h_{n}}\right| & \leq C_{0} \delta_{h_{n}}^{2}\left(\lambda_{i}\right) .
\end{aligned}
$$

Proof. We use induction to prove Theorem 8 .

First, for $n=1$, since $C\left(\lambda_{i}\right) \leq C_{0}$, we can obtain (86)-(88) from (84) and Lemma 2.

Suppose that (86)-(88) hold for $n-1$. Then from Theorem 5 we know that (86)-(88) are valid for $n$.

Corollary 9. Under the conditions of Theorem 8, further assume that $\left\{V_{h_{i}}\right\}_{i=1}^{n}$ is a series of linear or bilinear finite element spaces, and $M(\lambda) \subset H^{1+\sigma}(\Omega), r \leq \sigma \leq 1$. Then (86)-(88) are valid and

$$
\begin{gathered}
\eta_{a}(H) \leq C H^{r / 2}, \\
\delta_{h_{n}}\left(\lambda_{i}\right) \leq C h_{n}^{\sigma} .
\end{gathered}
$$

Remark 10. When $q=1$, we obtain error estimates (86) and (88) for simple eigenvalues. Comparing (86) and (88) with the estimates (4.6), (4.7) in [15], we know that our algorithm achieves the optimal convergence order and is as efficient as Algorithm 4.1 in [15].
Remark 11. With the iteration times increasing, the approximate eigenvalues are closer to the exact ones which lead the equation (32) more closer to singular, although it is not difficult to solve numerically (see [22]). However, we fix the shift in Algorithms 4 and 7 and get a new scheme. In Section 5, we will report a numerical experiment when the shift is fixed as $\tilde{\lambda}_{j, h_{k}}=\tilde{\lambda}_{j, h_{1}}$.

Remark 12. Reference $[15,17]$ analyzed that the computational costs of their algorithms are almost the same as those of solving the corresponding boundary value problem. Comparing our Algorithm 7 with Algorithm 7.2 in [15], it is not difficult to see that the computational work of these two algorithms are almost the same.

\section{Numerical Examples}

In this section, we consider the Steklov eigenvalue problem (1) on the unit square $\Omega=(-0.5,0.5)^{2}$ and the L-shape domain $\Omega=(0,2)^{2} \backslash[1,2]^{2}$, respectively. In our numerical experiments, we use algorithms in [15], our Algorithm 7, and Algorithm 7 with fixed shift $\tilde{\lambda}_{j, h_{k}}=\widetilde{\lambda}_{j, h_{1}}$ to solve (1), and the resulting approximations are denoted by $\lambda_{j, h_{k+1}}^{X}, \lambda_{j, h_{k+1}}^{S}$, and $\lambda_{j, h_{k+1}}^{F}$, respectively. Our programs are performed under the package of Chen (cf. [25]).

Example 1. We solve (1) on the unit square $\Omega=(-0.5,0.5)^{2}$ by three algorithms with the linear conforming element. Since the exact eigenvalues are unknown, we use $\lambda_{1} \approx$ 0.24007908542 and $\lambda_{2}=\lambda_{3} \approx 1.49230313453$ obtained by the spectral element method (see [28]) as the reference eigenvalues. We depict the error curves of three algorithms in Figure 1.

Example 2. We solve (1) on the L-shape domain $\Omega=$ $(0,2)^{2} \backslash[1,2]^{2}$ by three algorithms with the linear conforming element. Since the exact eigenvalues are unknown, we use $\lambda_{1} \approx 0.34141604255$ and $\lambda_{5} \approx 1.70092884408$ obtained by the spectral element method (see [28]) as the reference values. The error curves of three algorithms are depicted in Figure 2.

From Figures 1 and 2 we can see that our algorithm achieves the optimal convergence order and is as efficient as that in [15].

\section{Concluding Remark}

In this paper, we combined the correction technique proposed by Lin and Xie and the shifted inverse iteration to establish a new efficient multilevel correction scheme for the Steklov eigenvalue problem which is suitable not only for simple eigenvalues but also for multiple eigenvalues. The algorithm and analysis in this paper are suitable for the Steklov eigenvalue problem in three dimensional domains and also suitable for general second-order self-adjoint elliptic eigenvalue problems. 


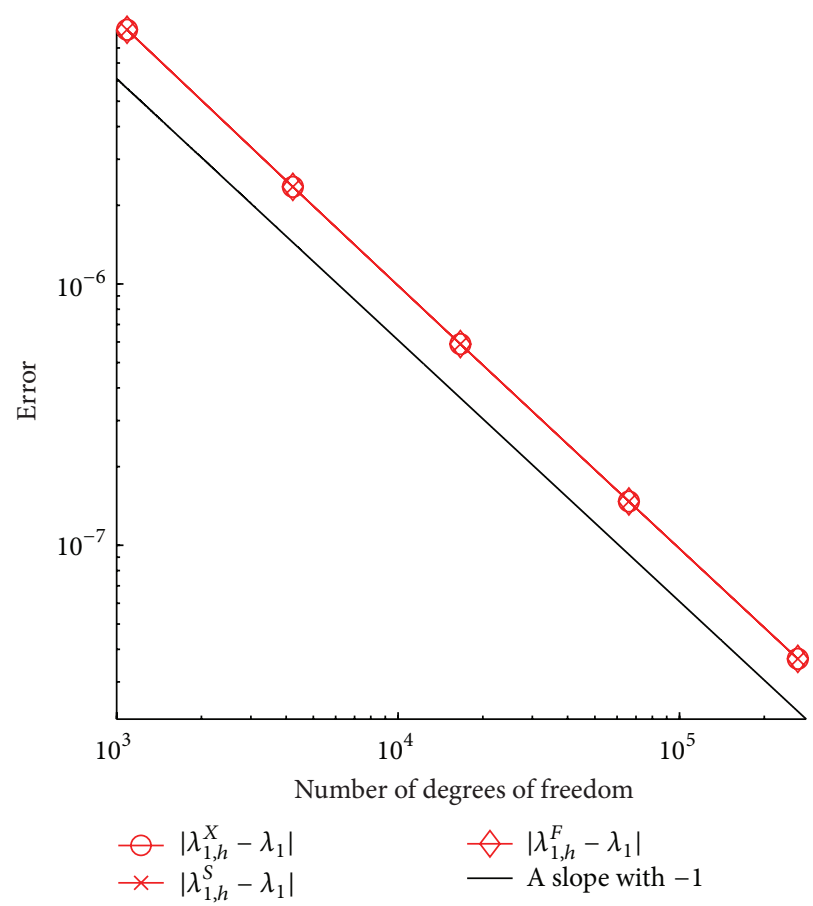

(a)

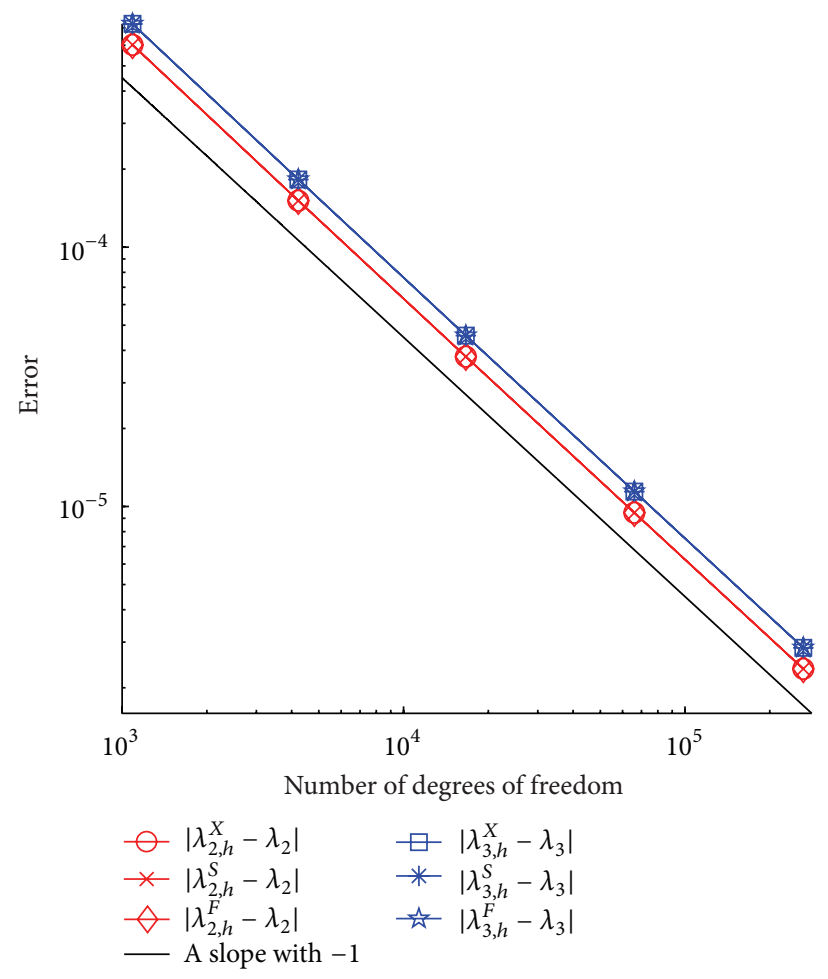

(b)

Figure 1: $\Omega=(-0.5,0.5)^{2}$, error curves for the 1st (a) and 2nd and 3rd (b) eigenvalues.

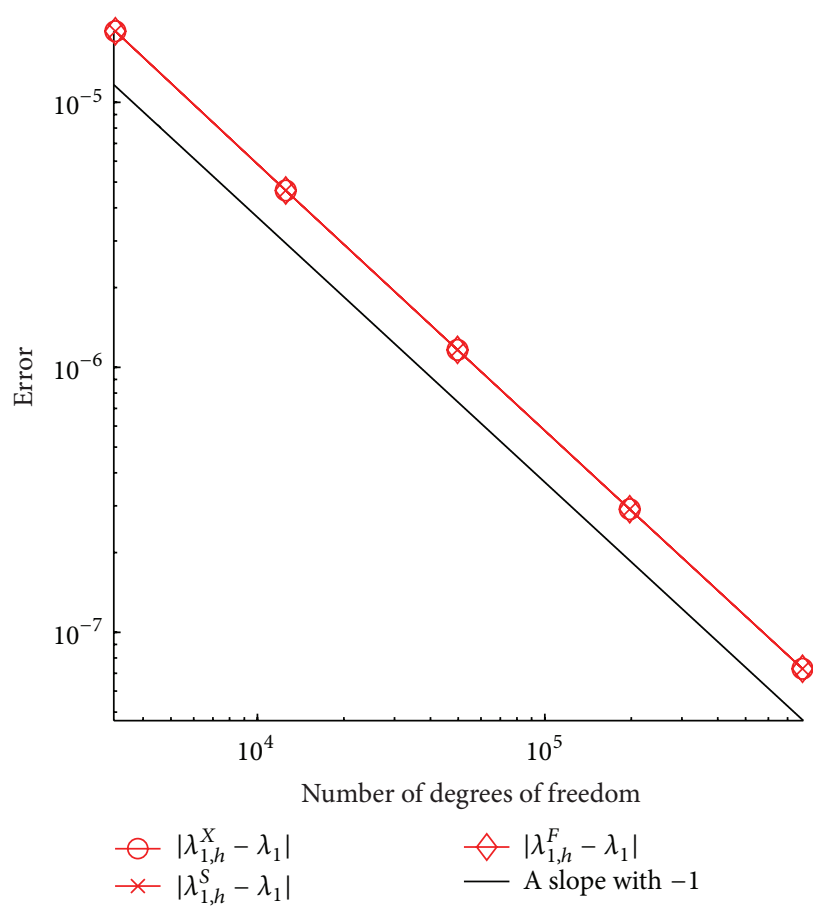

(a)

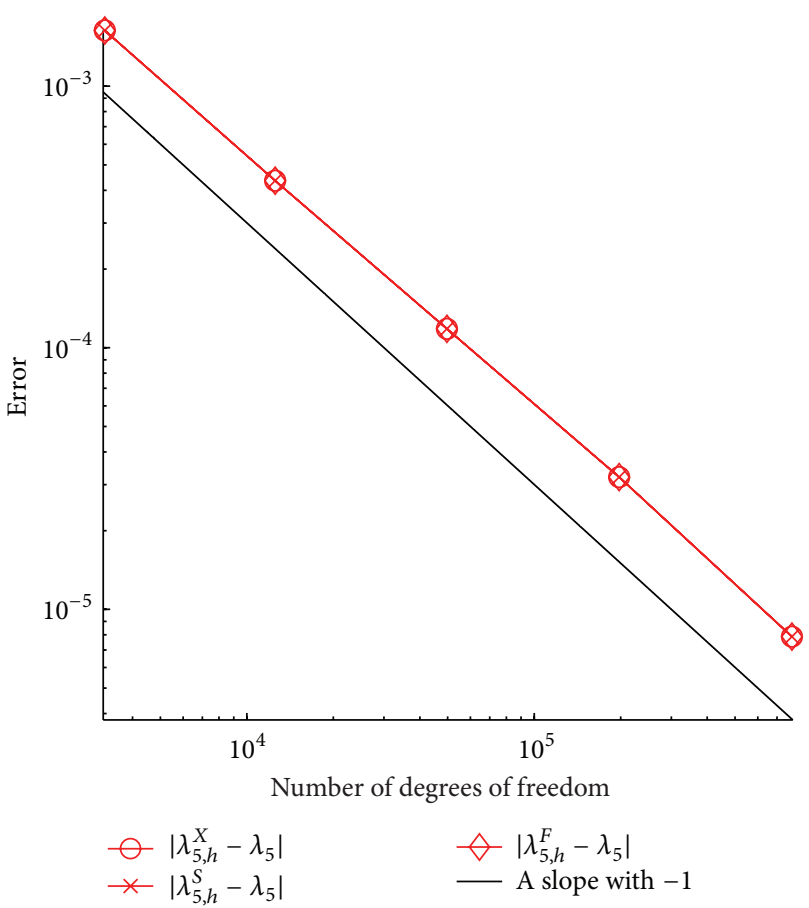

(b)

Figure 2: $\Omega=(0,2)^{2} \backslash[1,2]^{2}$, error curves for the 1st (a) and 5th (b) eigenvalues. 


\section{Conflict of Interests}

The authors declare that there is no conflict of interests regarding the publication of this paper.

\section{Acknowledgments}

This work is supported by National Natural Science Foundation of China (Grant no. 11201093). The authors cordially thank the editor and the reviewers for their valuable comments and suggestions and thank Jiayu Han for his help which lead to the improvement of the paper.

\section{References}

[1] S. Bergman and M. Schiffer, Kernel Functions and Elliptic Differential Equations in Mathematical Physics, Academic Press, New York, NY, USA, 1953.

[2] C. Conca, J. Planchard, and M. Vanninathan, Fluids and Periodic Structures, vol. 38, John Wiley \& Sons, Chichester, UK, 1995.

[3] A. Bermudez, R. Rodriguez, and D. Santamarina, "A finite element solution of an added mass formulation for coupled fluid-solid vibrations," Numerische Mathematik, vol. 87, no. 2, pp. 201-227, 2000.

[4] J. H. Bramble and J. E. Osborn, "Approximation of Steklov eigenvalues of non-selfadjoint second order elliptic operators," in The Mathematical Foundations of the Finite Element Method with Applications to Partial Differential Equations, pp. 387-408, Academic Press, New York, NY, USA, 1972.

[5] M. G. Armentano and C. Padra, "A posteriori error estimates for the Steklov eigenvalue problem," Applied Numerical Mathematics, vol. 58, no. 5, pp. 593-601, 2008.

[6] A. Dello Russo and A. E. Alonso, "A posteriori error estimates for nonconforming approximations of Steklov eigenvalue problems," Computers \& Mathematics with Applications, vol. 62, no. 11, pp. 4100-4117, 2011.

[7] Y. Yang and H. Bi, "Local a priori/a posteriori error estimates of conforming finite elements approximation for Steklov eigenvalue problems," Science China. Mathematics, vol. 57, no. 6, pp. 1319-1329, 2014.

[8] Q. Li, Q. Lin, and H. Xie, "Nonconforming finite element approximations of the Steklov eigenvalue problem and its lower bound approximations," Applications of Mathematics, vol. 58, no. 2, pp. 129-151, 2013.

[9] M. G. Armentano, C. Padra, R. Rodríguez, and M. Scheble, "An hp finite element adaptive scheme to solve the Laplace model for fluid-solid vibrations," Computer Methods in Applied Mechanics and Engineering, vol. 200, no. 1-4, pp. 178-188, 2011.

[10] H. Bi and Y. Yang, "A two-grid method of the non-conforming Crouzeix-Raviart element for the Steklov eigenvalue problem," Applied Mathematics and Computation, vol. 217, no. 23, pp. 9669-9678, 2011.

[11] H. Bi and Y. Yang, "Multiscale discretization scheme based on the rayleigh quotient iterative method for the Steklov eigenvalue problem," Mathematical Problems in Engineering, vol. 2012, Article ID 487207, 18 pages, 2012.

[12] E. M. Garau and P. Morin, "Convergence and quasi-optimality of adaptive FEM for Steklov eigenvalue problems," IMA Journal of Numerical Analysis, vol. 31, no. 3, pp. 914-946, 2011.
[13] Q. Li and Y. Yang, "A two-grid discretization scheme for the Steklov eigenvalue problem," Journal of Applied Mathematics and Computing, vol. 36, no. 1-2, pp. 129-139, 2011.

[14] M. Li, Q. Lin, and S. Zhang, "Extrapolation and superconvergence of the Steklov eigenvalue problem," Advances in Computational Mathematics, vol. 33, no. 1, pp. 25-44, 2010.

[15] H. Xie, "A type of multilevel method for the Steklov eigenvalue problem," IMA Journal of Numerical Analysis, vol. 34, no. 2, pp. 592-608, 2014

[16] Q. Lin and H. Xie, "A multi-level correction scheme for eigenvalue problems," Mathematics of Computation, vol. 84, no. 291, pp. 71-88, 2015.

[17] H. Xie, "A multigrid method for eigenvalue problem," Journal of Computational Physics, vol. 274, pp. 550-561, 2014.

[18] X. Ji, J. Sun, and H. Xie, "A multigrid method for Helmholtz transmission eigenvalue problems," Journal of Scientific Computing, vol. 60, no. 2, pp. 276-294, 2014.

[19] Z. Peng, H. Bi, H. Li, and Y. Yang, "A multilevel correction method for convection-diffusion eigenvalue problems," Mathematical Problems in Engineering, vol. 2015, Article ID 904347, 10 pages, 2015.

[20] T. Belytschko, R. Gracie, and G. Ventura, "A review of extended/ generalized finite element methods for material modeling," Modelling and Simulation in Materials Science and Engineering, vol. 17, no. 4, Article ID 043001, 24 pages, 2009.

[21] T.-P. Fries and T. Belytschko, "The extended/generalized finite element method: an overview of the method and its applications," International Journal for Numerical Methods in Engineering, vol. 84, no. 3, pp. 253-304, 2010.

[22] L. N. Trefethen and D. Bau, Numerical Linear Algebra, SIAM, Philadelphia, Pa, USA, 1997.

[23] Y. Yang and H. Bi, "Two-grid finite element discretization schemes based on shifted-inverse power method for elliptic eigenvalue problems," SIAM Journal on Numerical Analysis, vol. 49, no. 4, pp. 1602-1624, 2011.

[24] X. Hu and X. Cheng, "Acceleration of a two-grid method for eigenvalue problems," Mathematics of Computation, vol. 80, no. 275, pp. 1287-1301, 2011.

[25] L. Chen, "iFEM: an integrated finite element method package in MATLAB," Tech. Rep., University of California at Irvine, Irvine, Calif, USA, 2009.

[26] I. Babuska and J. Osborn, "Eigenvalue problems," in Handbook of Numerical Analysis, P. G. Lions and P. G. Ciarlet, Eds., vol. 2 of Finite Element Methods (Part 1), pp. 641-787, North-Holland, Amsterdam, The Netherlands, 1991.

[27] M. Dauge, Elliptic Boundary Value Problems on Corner Domains, vol. 1341 of Lecture Notes in Mathematics, Springer, Berlin, Germany, 1988.

[28] Y. J. Li, Y. D. Yang, and H. Bi, “The spectral element method for the Steklov eigenvalue problem," Advanced Materials Research, vol. 853, pp. 631-635, 2014. 


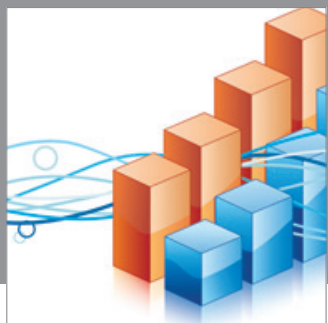

Advances in

Operations Research

mansans

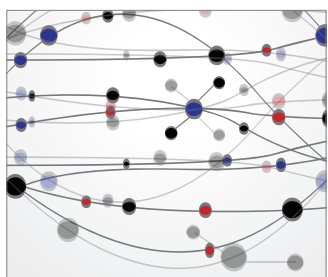

The Scientific World Journal
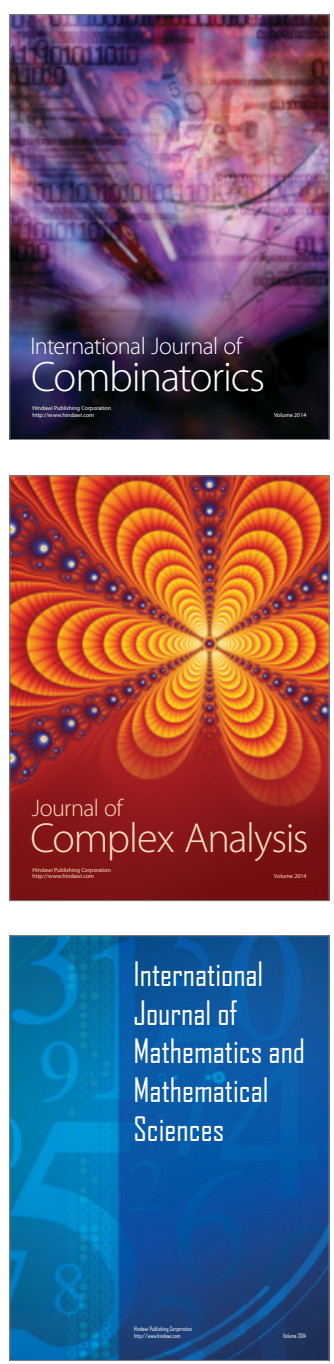
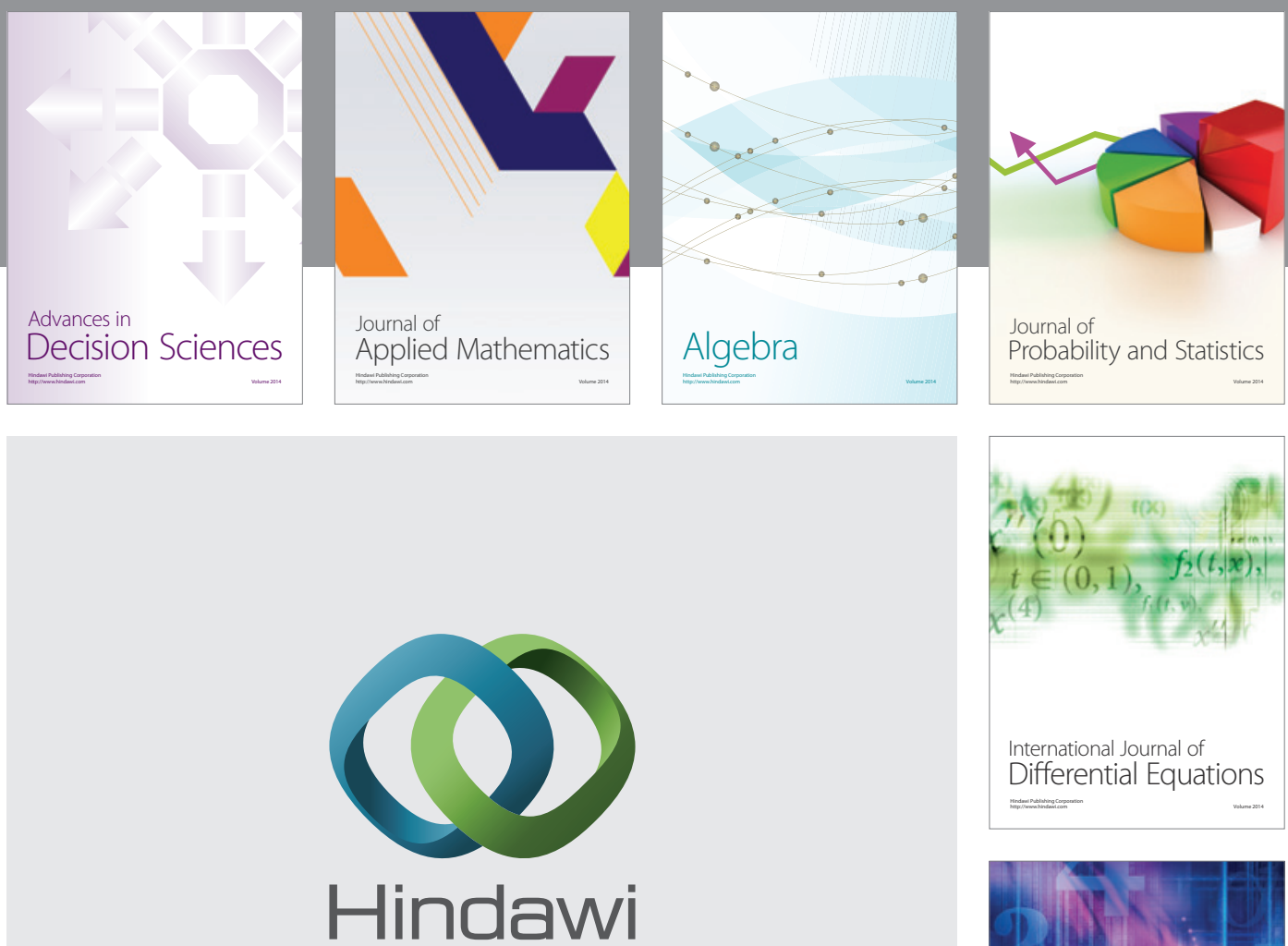

Submit your manuscripts at http://www.hindawi.com
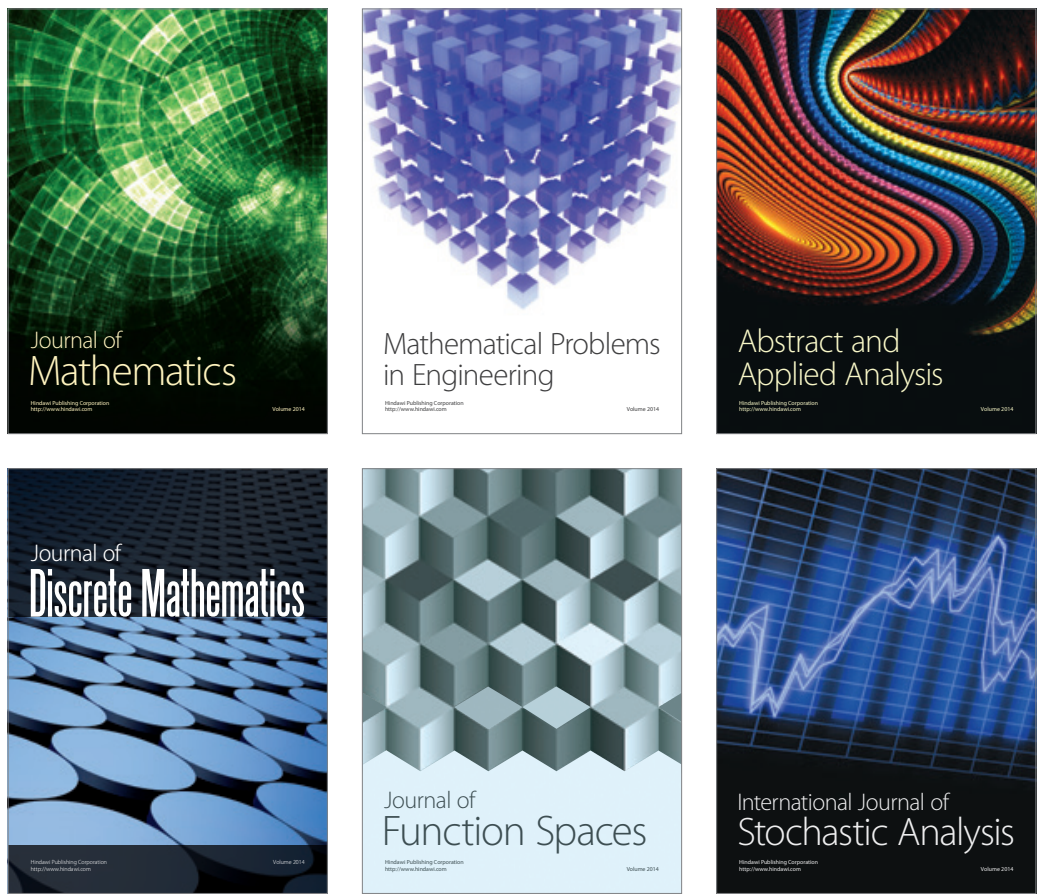

Journal of

Function Spaces

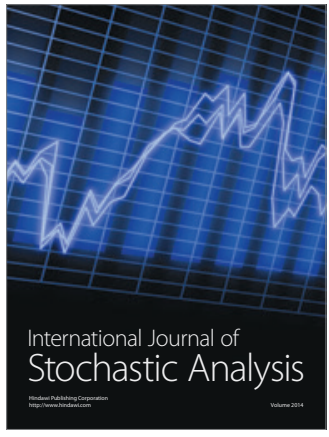

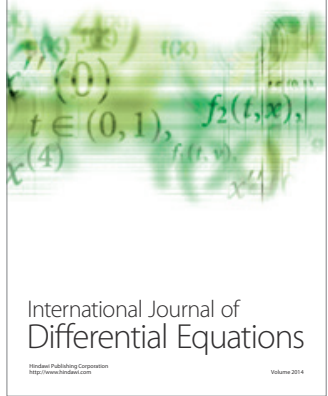
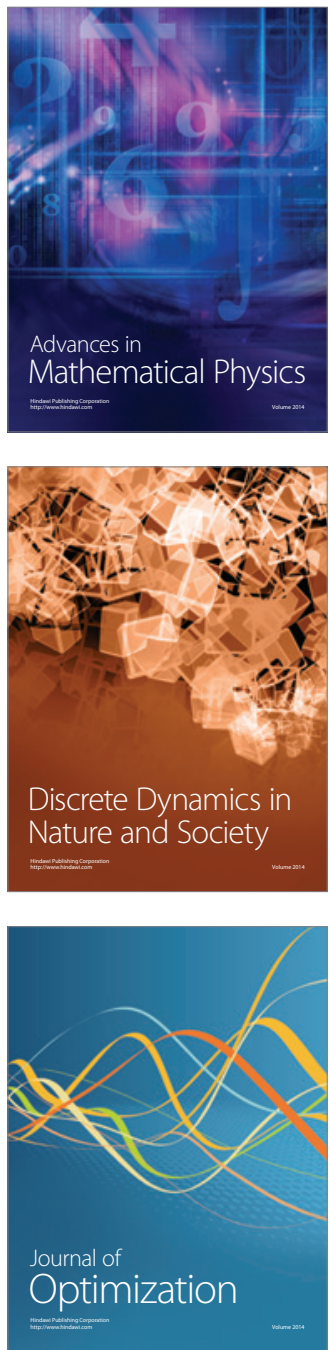